International Mathematical Forum, 2, 2007, no. 65, 3211 - 3218

\title{
Poisson Approximation for Independent Geometric Random Variables
}

\author{
K. Teerapabolarn ${ }^{1}$ and P. Wongkasem \\ Department of Mathematics, Faculty of Science \\ Burapha University, Chonburi 20131, Thailand
}

\begin{abstract}
The Stein-Chen method is used to derive two formulas of uniform and non-uniform bounds on Poisson approximation for a sum of $n$ independent geometric random variables. Application of these formulas is illustrated with the Poisson approximation to the negative binomial distribution.
\end{abstract}

Mathematics Subject Classification: Primary 60F05

Keywords: Geometric summands; negative binomial distribution; Poisson approximation; Stein-Chen method

\section{Introduction and main results}

In the past few years, there have been many papers on the Stein-Chen method for bounding Poisson approximation errors (see [3], [10] and references therein) and the application of the techniques to binomial, compound Poisson and geometric approximations: see [4], [2] and [6]. In this paper we further use the Stein-Chen method to bound error on Poisson approximation to the distribution of a sum of $n$ independent geometric random variables, which is thought of as the number of failures before the $n^{\text {th }}$ success in a sequence of Bernoulli trials. Before constructing such bound of the approximation, we define a random variable $X$ as the number of failures before the first success in a sequence of Bernoulli trials with probability of success $p, 0<p<1$. Thus $P(X=k)=(1-p)^{k} p, k=0,1, \ldots$, is the geometric probability with parameter $p$.

\footnotetext{
${ }^{1}$ Corresponding author, email : kanint@buu.ac.th
} 
Let $X_{1}, \ldots, X_{n}$ be $n$ independent geometric random variables with $P\left(X_{i}=\right.$ $k)=\left(1-p_{i}\right)^{k} p_{i}, k=0,1, \ldots$, but are not necessarily identical. Let $W=\sum_{i=1}^{n} X_{i}$ and $\lambda=E(W)=\sum_{i=1}^{n}\left(1-p_{i}\right) p_{i}^{-1}$, then $W$ is the number of failures before the $n^{\text {th }}$ success in a sequence of independent Bernoulli trials. If $p_{i}$ 's are identical to $p$, then $P(W=k)=\left(\begin{array}{c}n+k-1 \\ k\end{array}\right)(1-p)^{k} p^{n}, k=0,1, \ldots$, is the negative binomial distribution with parameters $n$ and $p$.

This paper we use the Stein-Chen method to determine two formulas of uniform and non-uniform error bounds for approximating the distribution of $W$ by the Poisson distribution with parameter $\lambda$. The following theorems are our main results.

Theorem 1.1. Let $W$ and $\lambda$ be defined as above. Then, for $A \subseteq \mathbb{N} \cup\{0\}$,

$$
\sup _{A}\left|P(W \in A)-\sum_{k \in A} \frac{\lambda^{k} e^{-\lambda}}{k !}\right| \leq \sum_{i=1}^{n} \min \left\{\frac{\lambda^{-1}\left(1-e^{-\lambda}\right)}{p_{i}}, 1\right\}\left(1-p_{i}\right)^{2} p_{i}^{-1} .
$$

Theorem 1.2. For $w_{0} \in \mathbb{N} \cup\{0\}$, the following formula holds:

$$
\left|P\left(W \leq w_{0}\right)-\sum_{k=0}^{w_{0}} \frac{\lambda^{k} e^{-\lambda}}{k !}\right| \leq \lambda^{-1}\left(e^{\lambda}-1\right) \sum_{i=1}^{n} \min \left\{\frac{1}{p_{i}\left(w_{0}+1\right)}, 1\right\}\left(1-p_{i}\right)^{2} p_{i}^{-1} .
$$

Remarks. 1. The error bounds in (1.1) and (1.2) are small when all $\left(1-p_{i}\right)$ are small, i.e. each result of the theorems yields a good Poisson approximation when all $\left(1-p_{i}\right)$ are small. Furthermore, the error bound in (1.2) depends on $w_{0}$, i.e. it is decreasing for $w_{0} \geq 0$.

2. In the case of $A=\{0\}$ or $w_{0}=0$, by applying Lemma 2.1 (3) of Teerapabolarn and Neammanee [11], we can obtain the following inequality

$$
\left|P(W=0)-e^{-\lambda}\right| \leq \lambda^{-2}\left(\lambda+e^{-\lambda}-1\right) \sum_{i=1}^{n}\left(1-p_{i}\right)^{2} p_{i}^{-1}
$$

\section{Proof of main results}

We will prove our main results by using the Stein-Chen method. The method was originally formulated for normal approximation by Stein in 1972, and it was adapted and applied to the Poisson case by Chen in 1975. This 
method started by Stein's equation for Poisson distribution with a parameter $\lambda$ which, given $h$, is defined by

$$
\lambda f(w+1)-w f(w)=h(w)-\mathcal{P}_{\lambda}(h),
$$

where $\mathcal{P}_{\lambda}(h)=e^{-\lambda} \sum_{k=0}^{\infty} h(k) \frac{\lambda^{k}}{k !}$ and $f$ and $h$ are bounded real valued functions defined on $\mathbb{N} \cup\{0\}$.

For $A \subseteq \mathbb{N} \cup\{0\}$, let $h_{A}$ be defined by

$$
h_{A}(w)= \begin{cases}1 & \text { if } w \in A \\ 0 & \text { if } w \notin A\end{cases}
$$

From Barbour et al. [3] on pp.7, the solution $f_{A}(w)$ of $(2.1)$ is of the form

$$
f_{A}(w)=\left\{\begin{array}{cl}
(w-1) ! \lambda^{-w} e^{\lambda}\left[\mathcal{P}_{\lambda}\left(h_{A \cap C_{w-1}}\right)-\mathcal{P}_{\lambda}\left(h_{A}\right) \mathcal{P}_{\lambda}\left(h_{C_{w-1}}\right)\right] & \text { if } w \geq 1 \\
0 & \text { if } w=0
\end{array}\right.
$$

where $C_{w}=\{0, \ldots, w\}$, and, for $w_{0} \in \mathbb{N} \cup\{0\}$, the solutions $f_{C_{w_{0}}}$ of $(2.1)$ can be expressed in the form of

$$
f_{C_{w_{0}}}(w)=\left\{\begin{array}{cl}
(w-1) ! \lambda^{-w} e^{\lambda}\left[\mathcal{P}_{\lambda}\left(h_{C_{w_{0}}}\right) \mathcal{P}_{\lambda}\left(1-h_{C_{w-1}}\right)\right] & \text { if } w_{0}<w \\
(w-1) ! \lambda^{-w} e^{\lambda}\left[\mathcal{P}_{\lambda}\left(h_{C_{w-1}}\right) \mathcal{P}_{\lambda}\left(1-h_{C_{w_{0}}}\right)\right] & \text { if } w_{0} \geq w \\
0 & \text { if } w=0
\end{array}\right.
$$

The following lemmas are established for proving the main results.

Lemma 2.1. Let $V f_{A}(w)=f_{A}(w+1)-f_{A}(w)$. Then, for $A \subseteq \mathbb{N} \cup\{0\}$ and $k \in \mathbb{N}$, we have

$$
\sup _{w \geq k}\left|V f_{A}(w)\right| \leq \min \left\{\lambda^{-1}\left(1-e^{-\lambda}\right), \frac{1}{k}\right\} .
$$

Proof. It is obtained from Barbour et al. [3] on pp.8.

Lemma 2.2. For $w_{0} \in \mathbb{N} \cup\{0\}$ and $k \in \mathbb{N}$, the following estimate holds:

$$
\sup _{w \geq k}\left|V f_{C_{w_{0}}}(w)\right| \leq \lambda^{-1}\left(e^{\lambda}-1\right) \min \left\{\frac{1}{w_{0}+1}, \frac{1}{k}\right\} \text {. }
$$

Proof. By using Lemma 2.1 (3) of Teerapabolarn and Neammanee [12], we have

$$
\sup _{w \geq k}\left|V f_{C_{w_{0}}}(w)\right| \leq \sup _{w \geq 1}\left|V f_{C_{w_{0}}}(w)\right| \leq \frac{\lambda^{-1}\left(e^{\lambda}-1\right)}{w_{0}+1} .
$$


Now we shall show that $\sup _{w \geq k}\left|V f_{C_{w_{0}}}(w)\right| \leq \frac{\lambda^{-1}\left(e^{\lambda}-1\right)}{k}$.

By the fact that

$V f_{C_{w_{0}}}(w)= \begin{cases}(w-1) ! \lambda^{-(w+1)} e^{-\lambda} \sum_{j=0}^{w_{0}} \frac{\lambda^{j}}{j !} \sum_{k=w+1}^{\infty}(w-k) \frac{\lambda^{k}}{k !}<0 & \text { if } w \geq w_{0}+1, \\ (w-1) ! \lambda^{-(w+1)} e^{-\lambda} \sum_{j=w_{0}+1}^{\infty} \frac{\lambda^{j}}{j !} \sum_{k=0}^{w}(w-k) \frac{\lambda^{k}}{k !}>0 & \text { if } 1 \leq w \leq w_{0} .\end{cases}$

For $w \geq w_{0}+1$ and $w \geq k$, by (2.7), we have

$$
\begin{aligned}
0<-V f_{C_{w_{0}}}(w) & \leq(w-1) ! \sum_{l=w+1}^{\infty}(l-w) \frac{\lambda^{l-(w+1)}}{l !} \\
& =(w-1) !\left\{\frac{1}{(w+1) !}+\frac{2 \lambda}{(w+2) !}+\cdots\right\} \\
& =\frac{(w-1) !}{w !}\left\{\frac{1}{w+1}+\frac{2 \lambda}{(w+1)(w+2)}+\cdots\right\} \\
& \leq \frac{1}{k} \lambda^{-1}\left\{\frac{\lambda}{2}+\frac{2 \lambda^{2}}{3 !}+\cdots\right\} \\
& \leq \frac{\lambda^{-1}\left(e^{\lambda}-1\right)}{k},
\end{aligned}
$$

and, for $k \leq w \leq w_{0}$,

$$
0<V f_{C_{w_{0}}}(w) \leq \frac{\lambda^{-1}\left(e^{\lambda}-1\right)}{w_{0}+1} \leq \frac{\lambda^{-1}\left(e^{\lambda}-1\right)}{k}
$$

Hence, (2.6) is obtained.

Proof of Theorem 1.1. We shall show that (1.1) holds.

From (2.1), given $h=h_{A}$, we have

$$
\left|P(W \in A)-\sum_{k \in A} \frac{\lambda^{k} e^{-\lambda}}{k !}\right|=|E[\lambda f(W+1)-W f(W)]|
$$

where $f=f_{A}$ is defined as in (2.3).

Note that

$$
|E[\lambda f(W+1)-W f(W)]| \leq \sum_{i=1}^{n}\left|E\left[\left(p_{i}^{-1}-1\right) f(W+1)-X_{i} f(W)\right]\right| .
$$


Let $W_{i}=W-X_{i}$. Then, for each $i$, we get

$$
\begin{aligned}
& E\left[\left(p_{i}^{-1}-1\right) f(W+1)-X_{i} f(W)\right] \\
& =E\left[\left(p_{i}^{-1}-1\right) f\left(W_{i}+X_{i}+1\right)-X_{i} f\left(W_{i}+X_{i}\right)\right] \\
& \left.=E\left[E\left[\left(p_{i}^{-1}-1\right) f\left(W_{i}+X_{i}+1\right)-X_{i} f\left(W_{i}+X_{i}\right)\right) \mid X_{i}\right]\right] \\
& \left.=E\left[\left(p_{i}^{-1}-1\right) f\left(W_{i}+X_{i}+1\right)-X_{i} f\left(W_{i}+X_{i}\right)\right) \mid X_{i}=0\right] P\left(X_{i}=0\right) \\
& \left.+E\left[\left(p_{i}^{-1}-1\right) f\left(W_{i}+X_{i}+1\right)-X_{i} f\left(W_{i}+X_{i}\right)\right) \mid X_{i}=1\right] P\left(X_{i}=1\right) \\
& \left.+\sum_{k \geq 2} E\left[\left(p_{i}^{-1}-1\right) f\left(W_{i}+X_{i}+1\right)-X_{i} f\left(W_{i}+X_{i}\right)\right) \mid X_{i}=k\right] P\left(X_{i}=k\right) \\
& \left.=E\left[\left(p_{i}^{-1}-1\right) f\left(W_{i}+X_{i}+1\right)-X_{i} f\left(W_{i}+X_{i}\right)\right) \mid X_{i}=0\right] p_{i} \\
& \left.+E\left[\left(p_{i}^{-1}-1\right) f\left(W_{i}+X_{i}+1\right)-X_{i} f\left(W_{i}+X_{i}\right)\right) \mid X_{i}=1\right] p_{i}\left(1-p_{i}\right) \\
& \left.+\sum_{k \geq 2} E\left[\left(p_{i}^{-1}-1\right) f\left(W_{i}+X_{i}+1\right)-X_{i} f\left(W_{i}+X_{i}\right)\right) \mid X_{i}=k\right] p_{i}\left(1-p_{i}\right)^{k} \\
& =E\left[\left(1-p_{i}\right) f\left(W_{i}+1\right)\right]+E\left[\left(1-p_{i}\right)^{2} f\left(W_{i}+2\right)-p_{i}\left(1-p_{i}\right) f\left(W_{i}+1\right)\right] \\
& +\sum_{k \geq 2} E\left[\left(1-p_{i}\right)^{k+1} f\left(W_{i}+k+1\right)-k p_{i}\left(1-p_{i}\right)^{k} f\left(W_{i}+k\right)\right] \\
& =\left[\left(1-p_{i}\right)-p_{i}\left(1-p_{i}\right)\right] E\left[f\left(W_{i}+1\right)\right]+E\left[\left(1-p_{i}\right)^{2} f\left(W_{i}+2\right)\right] \\
& +\sum_{k \geq 2} E\left[\left(1-p_{i}\right)^{k+1} f\left(W_{i}+k+1\right)-k p_{i}\left(1-p_{i}\right)^{k} f\left(W_{i}+k\right)\right] \\
& =\left(1-p_{i}\right)^{2} E\left[f\left(W_{i}+1\right)\right]+\sum_{k \geq 2} E\left[\left(1-p_{i}\right)^{k} f\left(W_{i}+k\right)-k p_{i}\left(1-p_{i}\right)^{k} f\left(W_{i}+k\right)\right] \\
& =\left(1-p_{i}\right)^{2} E\left[f\left(W_{i}+1\right)\right]+\sum_{k \geq 2} E\left[k\left(1-p_{i}\right)^{k} f\left(W_{i}+k\right)-k p_{i}\left(1-p_{i}\right)^{k} f\left(W_{i}+k\right)\right] \\
& -\sum_{k \geq 2} E\left[(k-1)\left(1-p_{i}\right)^{k} f\left(W_{i}+k\right)\right] \\
& =\left(1-p_{i}\right)^{2} E\left[f\left(W_{i}+1\right)\right]+\sum_{k \geq 2} E\left[k\left(1-p_{i}\right)^{k+1} f\left(W_{i}+k\right)\right] \\
& -\sum_{k \geq 2} E\left[k\left(1-p_{i}\right)^{k+1} f\left(W_{i}+k+1\right)\right]-\left(1-p_{i}\right)^{2} E\left[f\left(W_{i}+2\right)\right] \\
& =\left(1-p_{i}\right)^{2} E\left[f\left(W_{i}+1\right)-f\left(W_{i}+2\right)\right]+\sum_{k \geq 2} E\left[k\left(1-p_{i}\right)^{k+1} f\left(W_{i}+k\right)\right] \\
& -\sum_{k \geq 2} E\left[k\left(1-p_{i}\right)^{k+1} f\left(W_{i}+k+1\right)\right] \\
& =\left(1-p_{i}\right)^{2} E\left[f\left(W_{i}+1\right)-f\left(W_{i}+2\right)\right] \\
& +\sum_{k \geq 2} k\left(1-p_{i}\right)^{k+1} E\left[f\left(W_{i}+k\right)-f\left(W_{i}+k+1\right)\right] \\
& =\sum_{k \geq 1} k\left(1-p_{i}\right)^{k+1} E\left[f\left(W_{i}+k\right)-f\left(W_{i}+k+1\right)\right]
\end{aligned}
$$


By using Lemma 2.1,

$$
\begin{aligned}
\mid E\left[\left(p_{i}^{-1}-1\right) f(W+1)\right. & \left.-X_{i} f(W)\right] \mid \\
& \leq \sum_{k \geq 1} k\left(1-p_{i}\right)^{k+1} E\left|f\left(W_{i}+k\right)-f\left(W_{i}+k+1\right)\right| \\
& \leq \sum_{k \geq 1} k\left(1-p_{i}\right)^{k+1} \sup _{w \geq k}|V f(w)| \\
& \leq \min \left\{\lambda^{-1}\left(1-e^{-\lambda}\right) \sum_{k \geq 1} k\left(1-p_{i}\right)^{k+1}, \sum_{k \geq 1}\left(1-p_{i}\right)^{k+1}\right\} \\
& =\min \left\{\lambda^{-1}\left(1-e^{-\lambda}\right)\left(1-p_{i}\right)^{2} p_{i}^{-2},\left(1-p_{i}\right)^{2} p_{i}^{-1}\right\} \\
& =\min \left\{\frac{\lambda^{-1}\left(1-e^{-\lambda}\right)}{p_{i}}, 1\right\}\left(1-p_{i}\right)^{2} p_{i}^{-1} .
\end{aligned}
$$

Hence, by (2.8), (2.9) and (2.10), (1.1) holds.

Proof of Theorem 1.2. Using the same argument detailed as in the proof of Theorem 1.1 together with Lemma 2.2, the theorem is obtained.

\section{An illustrative application}

This section gives an illustrative application of the theorems to approximate the negative binomial by the Poisson distribution with parameter $\lambda=$ $n p^{-1}(1-p)$ in two formulas. Finally, we compare our result, a uniform bound, with some other results.

\subsection{Uniform and non-uniform bounds}

When $p_{i}$ 's are identical to $p$, we have $\lambda=n p^{-1}(1-p)$ and a uniform bound for approximating the negative binomial distribution by the Poisson distribution in Theorem 1.1 is of the form

$$
\sup _{A}\left|P(W \in A)-\sum_{k \in A} \frac{\lambda^{k} e^{-\lambda}}{k !}\right| \leq \min \left\{1-e^{-\lambda}, \lambda p\right\} \frac{1-p}{p}
$$

where $A \subseteq \mathbb{N} \cup\{0\}$. For $w_{0} \in \mathbb{N} \cup\{0\}$, a non-uniform bound in Theorem 1.2 is in the form of

$$
\left|P\left(W \leq w_{0}\right)-\sum_{k=0}^{w_{0}} \frac{\lambda^{k} e^{-\lambda}}{k !}\right| \leq \min \left\{\frac{1}{w_{0}+1}, p\right\}\left(e^{\lambda}-1\right) \frac{1-p}{p}
$$


and in the case of $w_{0}=0$, by (1.3), we have

$$
\begin{aligned}
\left|P(W=0)-e^{-\lambda}\right| & \leq \lambda^{-1}\left(\lambda+e^{-\lambda}-1\right)(1-p) \\
& =\frac{\left(\lambda+e^{-\lambda}-1\right) p}{n} .
\end{aligned}
$$

It should be noted that the bounds in (3.1) and (3.2) are small when $1-p$ is small or $\lambda$ is small, i.e. each result of (3.1) and (3.2) gives a good Poisson approximation for small $1-p$ or small $\lambda$. Moreover, the bound in (3.2) can be decreased by increasing $w_{0}$.

\subsection{Comparison with some other results}

We will compare our result of (3.1) with some other results below.

$$
\begin{aligned}
& \sup _{A}\left|P(W \in A)-\sum_{k \in A} \frac{\lambda^{k} e^{-\lambda}}{k !}\right| \leq \frac{1-p}{p} \quad \text { (Vervaat [13]), } \\
& \sup _{A}\left|P(W \in A)-\sum_{k \in A} \frac{\lambda^{k} e^{-\lambda}}{k !}\right| \leq \frac{1-p}{\sqrt{2} p} \quad \text { (Romanowska [8]), } \\
& \sup _{A}\left|P(W \in A)-\sum_{k \in A} \frac{\lambda^{k} e^{-\lambda}}{k !}\right| \leq \lambda(1-p) \quad \text { (Gerber [5]), } \\
& \sup _{A}\left|P(W \in A)-\sum_{k \in A} \frac{\lambda^{k} e^{-\lambda}}{k !}\right| \leq \frac{\lambda(1-p)}{p} \quad \text { (Pfeifer [7]), } \\
& \sup _{A}\left|P(W \in A)-\sum_{k \in A} \frac{\lambda^{k} e^{-\lambda}}{k !}\right| \leq \frac{\left(1-e^{-\lambda}\right)(1-p)}{p} \quad \text { (Barbour [1]), } \\
& \sup _{A}\left|P(W \in A)-\sum_{k \in A} \frac{\lambda^{k} e^{-\lambda}}{k !}\right| \leq \min \left\{\frac{3}{4 e}, \lambda\right\} \frac{1-p}{p} \quad \text { (Roos [9]). }
\end{aligned}
$$

Note that (3.8) can also be derived from Theorem 1.C (ii) of Barbour et al. [3]. Since our bound, the bound in (3.1), is the minimum value of the bounds in (3.6) and (3.8), thus it is better than the bounds in (3.4) and (3.7). If $1-p<\frac{1}{\sqrt{2} n}$ or $\lambda<-\log \left(1-\frac{1}{\sqrt{2}}\right)$, then our bound is better than Romanowska's bound (see (3.5)). Observe that Roos's bound (see (3.9)) is better than Romanowska's bound, hence the bound in (3.1) is better than the bounds in (3.5) and (3.9) if $1-p<\frac{3}{4 e n}$ or $\lambda<-\log \left(1-\frac{3}{4 e}\right)$.

\section{Acknowledgements}

The authors would like to thank Faculty of Science, Burapha University, for financial support. 


\section{References}

[1] A.D. Barbour, Asymptotic expansions in the Poisson limit theorem, Ann. Prob., 15 (1987), 748-766.

[2] A.D. Barbour, L.H.Y. Chen, W.L. Loh, Compound Poisson approximation for nonnegative random variables via Stein's method, Ann. Prob., 20 (1992), 1843-1866.

[3] A.D. Barbour, L. Holst, S. Janson, Poisson approximation, Oxford Studies in Probability 2, Clarendon Press, Oxford, 1992.

[4] W. Ehm, Binomial approximation to the Poisson binomial distribution, Statist. Prob. Lett., 11 (1991), 7-16.

[5] H.U. Gerber, Error bounds for the compound Poisson approximation, Insurance Math. Econom., 3 (1984), 191-194.

[6] E. Pekoz, Stein's method for geometric approximation, J. Appl. Prob., 33 (1996), 707-713.

[7] D. Pfeifer, On the distance between mixed Poisson and Poisson distributions, Statist. Decisions., 5 (1987), 367-379.

[8] M. Romanowska, A note on the upper bound for the distance in total variation between the binomial and the Poisson distributions, Statist. Neerlandica., 31 (1977), 127-130.

[9] B. Roos, Improvements in the Poisson approximation of mixed Poisson distributions, J. Statist. Plann. Inference, 113 (2003), 467-483.

[10] C.M. Stein, Approximate Computation of Expectations, IMS, Hayward California, 1986.

[11] K. Teerapabolarn, K. Neammanee, A non-uniform bound on Poisson approximation in somatic cell hybrid model, Math. BioSc., 195 (2005), 5664.

[12] K. Teerapabolarn, K. Neammanee, Poisson approximation for sums of dependent Bernoulli random variables, Acta Math., 22 (2006), 87-99.

[13] W. Vervaat, Upper bound for distance in total variation between the binomial or negative binomial and the Poisson distribution, Statist. Neerlandica., 23 (1969), 79-86.

Received: June 27, 2007 